\title{
A TEORIA GERAL DE PROBLEMAS VARIACIONAIS LINEARES APROXIMADOS: APLICAÇÃO À ANÁLISE DE ERRO DO MÉTODO DOS VOLUMES FINITOS
}

Paulo R. Trales

\section{RESUMO}

A teoria geral de aproximação de problemas variacionais lineares desenvolvida por BABUSKA no início dos anos setenta, e refinada por DUPIRE, em sua tese defendida na PUC-Rio em 1985, é, juntamente com as estimativas clássicas do erro da interpolação polinomial em espaços de Sobolev, o ingrediente básico para a análise de convergência de soluções aproximadas de equações diferenciais pelo Método dos Elementos Finitos. O objetivo deste trabalho é mostrar que ambas são também ferramentas apropriadas para as análises de erro e convergência do Método dos Volumes Finitos. Mais especificamente, depois de recapitular os resultados de DUPIRE, no que tange à aproximação abstrata dessa classe de problemas, damos um exemplo de estimativa de erro aplicada ao método dos volumes finitos, que ilustra tal asserção.

\section{INTRODUÇÃO}

A teoria matemática de aproximação dos métodos de elementos finitos é hoje bem estabelecida, graças notadamente aos estudos conduzidos por diversos autores, no final da década de sessenta e no início da de setenta. Entre as contribuições decisivas para se chegar a esses resultados fundamnetais, citaríamos os trabalhos de AUBIN [1], AZIZ \& BABUSKA [2], BRAMBLE [5], CIARLET \& RAVIART [6], STRANG [10], entre outros.

Esses primeiros estudos focalizaram primordialmente problemas elíticos, onde a coercividade da forma bilinear contínua do problema variacional subjacente, permite reduzir a estimativa do erro cometido - pelo menos na ausência de crimes variacionais [10] sà do erro da interpolação polinomial medido em normas de espaços de Sobolev. No intuito de recapitular brevemente tais mecanismos, consideremos:

- $\quad Y$ um espaço de Hilbert de norma $\|\cdot\|_{Y}$;

- $\quad L$ uma forma linear contínua sobre $Y$ (i.e. $L \in Y^{\prime}$, o dual topológico de $Y$ );

- $\quad a: Y \times Y \rightarrow \mathbb{R}$ uma forma bilinear contínua. 
No intuito de abreviar o texto, usaremos daqui para a frente a notação dada na

Definição 1.1. Sejam $X$ e $Y$ dois espaços vetoriais normados respectivamente por $\|\cdot\|_{X} e\|\cdot\|_{Y} \cdot L_{2 c}(X \times Y)$ é definido como o espaço das formas $a: X \times Y \rightarrow I R$ que são bilineares e continuas.

Lembremos que $a \in L_{2 c}(X \times Y)$ se e somente se existe uma constante $M$ tal que

$$
a(x, y) \leq M\|x\|_{X}\|y\|_{Y}, \forall x \in X, y \in Y .
$$

Aliás neste caso define-se a norma de $a$, como ínfimo das constantes $M$ que verificam a relação acima, ou seja:

$$
\|a\|=\sup _{\left\{\begin{array}{l}
(x, y) \in X \times Y \\
(x, y) \neq 0
\end{array}\right.} \frac{a(x, y)}{\|x\|_{X}\|y\|_{Y}} .
$$

Seja agora o problema variacional linear:

$$
\left(P_{Y}\right)\left\{\begin{array}{l}
\text { Encontrar } x \in Y \\
a(x, y)=L(y) \quad \forall y \in Y .
\end{array}\right.
$$

O clássico Teorema de Lax-Milgram garante que se $a$ for $Y$-elítica, ou equivalentemente, coerciva sobre $Y \times Y$, o que significa que:

$$
\exists \alpha>0 \text { tal que } a(y, y) \geq \alpha\|y\|_{Y}^{2}, \forall y \in Y .
$$

então $\left(P_{Y}\right)$ admite uma solução única para qualquier $L$. Mais ainda, neste caso, se aproximarmos $\left(P_{Y}\right)$ por $\left(P_{Z}\right)$, deduzido de $\left(P_{Y}\right)$ mediante a substituição de $Y$ por um seu subespaço $Z$ (tipicamente de dimensão finita), e a solução $x \in Y$ por sua aproximação $\varpi \in Z$. então a distância entre $x$ e $\varpi$ pode ser estimada usando-se a desigualdade abaixo. resultado conhecido como o Lema de Céa:

$$
\|x-\varpi\|_{Y} \leq \frac{\|a\|}{\alpha} d_{Y}(x, Z),
$$

onde $d_{Y}(y, Z)$ que designa a distância de um elemento $y \in Y$ ao subespaço $Z$ medida na norma de $Y$, é dada por:

$$
d_{Y}(y, Z)=\inf _{z \in Z}\|y-z\|_{Y} .
$$

No início dos anos setenta, no intuito de estudar alguns problemas particulares, e notadamente problemas de ponto-sela, que não apresentam as características de $Y$ eliticidade, vale dizer de coercividade, alguns autores, entre eles BABUSKA [4], consideraram a extensão do Teorema de Lax-Milgram ao problema seguinte, mais 
geral do que $\left(P_{Y}\right)$, no qual a solução pode ser procurada num outro espaço de Hilbert $X$ de norma $\cdot{ }_{X}:$

$$
\left(P_{X, Y}\right)\left\{\begin{array}{lr}
\text { Encontrar } x \in X & \text { ta l que } \\
a(x, y)=L(y) & \forall y \in Y .
\end{array}\right.
$$

Aquí se supõe naturalmente que $a: X \times Y \rightarrow \mathbb{R}$ é bilinear e contínua segundo a definição (1). Neste caso vale o seguinte resultado, conhecido como o Teorema de Lax-Milgram generalizado:

Teorema 1.1. Sob as Hipóteses $a \in L_{2 c}(X \times Y)$ e $L \in Y^{\prime}$, se a verifica as duas condições abaizo:

$$
\begin{aligned}
& \exists \alpha_{1}>0 \text { tal que } \forall x \in X \sup _{\substack{y \in Y \\
y \neq 0}} \frac{a(x, y)}{\|y\|_{Y}} \geq \alpha_{1}\|x\|_{X},
\end{aligned}
$$

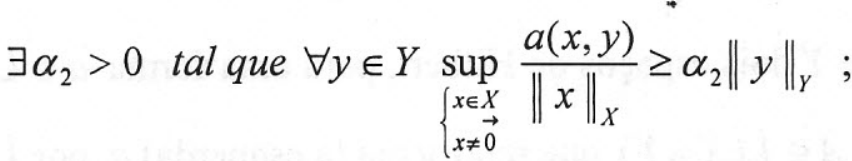

o problema $\left(P_{X, Y}\right)$ admite uma solução única para qualquer $L \in Y^{\prime}$.

Definição 1.2. Uma forma bilinear a que verifica as duas condições (7) e (8) é dita fracamente coerciva.

Observe-se que no caso em que $X=Y$, o fato de a ser coerciva implica que a é também fracamente coerciva, podendo-se tomar $\alpha_{1}=\alpha_{2}=\alpha$, onde $\alpha$ é a constante da desigualdade (3). Mais ainda, conforme demonstrado por DUPIRE [7], quando a é fracamente coerciva. na condição (8) pode-ser tomar $\alpha_{2}=\alpha_{1}=\alpha$, e reciprocamente, sendo $\alpha$ a maior das constantes com as quais se verificaram as duas desigualdades. Isto enseja uma aproximação ainda maior dos conceitos de coercividade e de fraca coercividade, posto que as duas condições acima podem ser vistas como uma extensão direta daquela que caracteriza a coercividade. Na realidade, não é só nesse aspecto que uma analogia se apresenta: daremos neste trabalho uma recapitulação da análise feita por PִUPIRE [7], segundo a qual uma desigualdade análoga à do Lema de Céa vale para o caso em que $a$ é fracamente coerciva, com uma constante dependente de $a$, obtida mediante a simples substitução em (4) da constante $\alpha$ que caracteriza a coercividade, pela (agora única constante que expressa a fraca coercividade de $a$ relativamente ao problema que aproxima $\left(P_{X, Y}\right)$. Na Seção seguinte vamos examinar 
mais em detalhes essa asserção, na medida em que essa analogia não fica transparente em trabalhos mais conhecidos sobre o assunto, como os de BABUSKA já citados. Por exemplo, considera-se nos mesmos que a majoração de erro no caso fracamente coercivo é válida com uma constante igual a um mais a constante obtida da forma acima indicada.

Antes de passarmos a essa análise, completamos a notação e recapitulamos alguns fatos e conceitos abaixo:

- Sendo $Y$ um espaço de Hilbert denotamos seu produto interno por $(\cdot, \cdot)_{Y}$ associado à norma $\|\cdot\|_{Y}$;

- Sejam $V$ e $Z$ dois subespaços fechados de $Y$ tais $Y=V \oplus Z$ (soma direta mas não necessariamente ortogonal). Define-se o operador de projeção sobre $V$ paralelamente a $Z$ (resp. Projeção sobre $Z$ paralelamente a $V$ ), denotada por $P=\pi_{V \| / Z} \quad$ (resp. por $Q=\pi_{Z \| V}=I-P$ ), pela relação: Dado $y \in Y$, $\left(P_{y}, v\right)_{Y}=(y, v)_{,} \quad \forall v \in V$ (resp. $\left.\left(Q_{y}, z\right)_{Y}=(y, z)_{Y} \quad \forall z \in Z\right)$;

- Sendo $X$ e $Y$ dois espaços de Hilbert, para uma forma $a \in L_{2 c}(X \times Y)$, define-se o operador $A \in L(X \times Y)$ que representa (à esquerda) $a$, por $(A(x), y)_{Y}=a(x, y)$;

- A norma padrão de $A$ acima no espaço $L(X \times Y)$ denotada por $\|A\|$, é igual a $\|a\|$

- Para uma forma $a \in L_{2 c}(X \times Y)$, define-se a conorma do operador $A \in L(X \times Y)$ que a representa (à esquerda), denotada conorma $(A)$ por

$$
\operatorname{conorma}(A)=\sup _{\left\{\begin{array}{c}
x \in X \\
x \neq 0
\end{array}\right.} \frac{\|A(x)\|_{Y}}{\|x\|_{X}} .
$$

Aqui se observa que necesariamente $\operatorname{conorma}(A) \geq 0$, e que somente se a condição (7) é verificada temos c'onorma $(A)>0$ (sendo conorma $(A) \geq \alpha_{1}$ ). Além disso, conforme observado por DUPIRE [7], ambas as condições (7) e (8) são também necessárias para que $\left(P_{X, Y}\right)$ seja bem posto para qualquer $L \in L^{\prime}$. Deduz-se assim por uma trivial aplicação do Teorema de Representação de Riesz, que elas equivalem à bijetividade de $A$.

Enfim notamos que ambos operadores $P$ e $Q$ definidos acima pertencem a $L(X \times Y)$. 


\section{PROBLEMAS VARIACIONAIS LINEARES SOBRE SUBESPAÇOS}

Consideremos de agora em diante que, no intuito de resolver aproximadamente o problema variacional linear $\left(P_{X, Y}\right)$, definimos dois subespaços fechados $W$ e $Z$ respectivamente de $X$ e $Y$. Isto leva a postular o problema $\left(P_{W, Z}\right)$ definido da mesma forma que $\left(P_{X, Y}\right)$, mediante a substitução de $X$ por $W$ e de $Y$ por $Z$, e da solução $x \in X$ por $\varpi \in W$ destinada a aproximar a primeira.

Podemos definir as restrições $a_{W, Z}$ de $a$ a $W \times Z$ e $L_{Z}$ de $L$ a $Z$ por

$$
\begin{gathered}
a_{W, Z} \in L_{2 c}(W \times Z): \forall(\varpi, z) \in W \times Z, a_{W, Z}(\varpi, z)=a(\varpi, z) \\
L_{Z} \in Z^{\prime}: \forall z \in Z \quad L_{Z}(z)=L(z) .
\end{gathered}
$$

Sabemos que existen $A_{W, Z} \in L(W, Z)$ e $f_{Z} \in Z$ tais que

$$
\forall(\varpi, z) \in W \times Z, a_{W, Z}(\varpi, z)=\left(A_{W, Z}(\varpi), z\right)_{Y}
$$

e

$$
\forall z \in Z \quad L_{Z}(z)=\left(f_{Z}, z\right)_{Y} .
$$

Podemos exprimir $A_{\| \prime Z}$ e $f_{Z}$ em função de $A$ e $f$ da seguinte forma:

Seja $\pi_{z}$ o operador de projeção ortogonal sobre $Z$. Temos $\forall(\varpi, z) \in W \times Z$, $\left(A_{W, Z}(\varpi), z\right)_{Y}=(A(\varpi), z)_{Y}$ ou seja $\left(A(\varpi)-A_{W, Z}(\varpi), z\right)_{Y}=0 \quad \forall \varpi \in W \quad$ e $\forall z \in Z$. Logo $\left(A-A_{W, Z}\right)(\varpi) \in Z^{\perp}$, isto é, $A_{W, Z}(\varpi)=\pi_{Z} A(\varpi)$ para qualquer $\varpi \in W$. Por conseguinte,

$$
A_{W, Z}=\pi_{Z} A_{\mid W}
$$

ou seja, $A_{W, Z}$ é a restrição a $W$ de $A$ composta com a projeção ortogonal sobre $Z$. Além disso, segundo o Teorema de Lax-Milgram generalizado, o problema $\left(P_{W, Z}\right)$ é bem posto para todo $L$ em $Y^{\prime}$ se e somente se $a_{W, Z}$ é fracamente coerciva, pois verifica-se facilmente que as restrições de $L$ a $Z$ descrevem $Z^{\prime}$.

Observação 2.1. Uim fato importante de se salientar, é que, ao contrário da coercìidade, a fracu coercividade não se transmite aos subespaços, o que implica aqui que, para cada par de subespaços selecionados para definir o problema aproximado, temos que verificar que $\left(P_{W, Z}\right)$ é bem posto, vale dizer, que a forma a (ou sua restrição) é fracamente coerciva sobre $W \times Z$, ou equivalentemente, que o 
operador $A_{W, Z}=\pi_{Z} \cdot A_{\mid W}$ é uma bijeção entre $W$ e $Z$, Assim, por abuso de linguagem, diremos que neste caso $A_{W, Z}$ é fracamente coercivo sobre $W \times Z$.

Aliás, justamente para simplificar a linguagem e as notações, damos as definições seguintes:

Definição 2.1. Seja $A \in L(X \times Y)$ e sejam $W \subset X$ e $Z \subset Y$ dois subespaços fechados. $A$ é dito $(W, Z)$ f.c. se $\pi_{Z} A_{W}$ é fracamente coercivo, isto é pertence a $\operatorname{Isom}_{c}(W, Z)$. Além disso, neste caso a conorma de $\pi_{Z} A_{\mid W}$ é denotada por $\alpha_{W, Z}$.

Essas definições são transferidas trivialmente $a \in L_{2 c}(X \times Y)$. Aplicamo-las imediatamente no seguinte contexto (cf. [7]):

Proposição 2.1. Se $a \in L_{2 c}(X \times Y)$ é $(W, Z)$ f.c. então $W \oplus \operatorname{Ker} \pi_{Z} A=X$, onde $A \in L(X \times Y)$ representa (à esquerda) a.

DEMOSTRAÇÃO. Seja $\varpi \in W \cap K e r \pi_{z} A$, isto é $A_{W, Z} \varpi=\pi_{Z} A_{\mid W} \varpi=0$. Logo $W \cap \operatorname{Ker} \pi_{Z} A=\{0\}$, pois $A_{W, Z}$ é injetivo.

Agora seja $x \in X$ e $x_{W} \in W$ a solução única (pois $A$ é $(W, Z) f$.c.) do problema variacional seguinte: Achar $x_{W} \in W$ tal que, $\forall z \in Z, a\left(x_{W}, z\right)$. Observamos que $x-x_{W} \in \operatorname{Ker} \pi_{Z} A$. Com efeito, $\forall z \in Z,\left(A\left(x-x_{W}\right), z\right)_{Y}=a\left(x-x_{W}, z\right)=0$.

Logo a descomposição $x=x_{W}+\left(x-x_{W}\right)$ mostra que $W \oplus \operatorname{Ker} \pi_{Z} A=X$.

O Lema seguinte devido a DUPIRE (cf. [7], Proposição II-3.3) fornece um resultado fundamental para se obter uma estimativa fina e simples de $\left\|x-x_{W}\right\|_{X}$ em relação a $d_{X}(x, W)=\left\|x-\pi_{W} x\right\|_{X}$ na Proposição 2.1 dada mais adiante (cf. [7]).

Lema 2.1. Sejam $A \in L(X \times Y), W \subset X$ e $Z \subset Y$, W e $Z$ subespaços fechados.

Sejam ainda $A_{1}=\pi_{Z} A_{\mid W} \in L_{c}(W, Z)$ e $A_{2}=\pi_{Z} A_{\mid W \perp}$. Então $A_{1}$ ser sobrejectivo implica:

$$
\therefore \quad\left|\operatorname{conorma}\left(A_{1}\right)\right|^{2}+\left\|A_{2}\right\|^{2} \leq\left\|\pi_{\mathrm{w}} A\right\|^{2} \leq\|A\|^{2} .
$$

Estamos agora em condições de postular a:

Proposição 2.2. Seja $a \in L_{2 c}(X \times Y)$ tal que a é $(W, Z)$ f.c., onde $W$ e $Z$ subespaços fechados de $X$ e $Y$. Então, para qualquer $x \in X$ : 
a) $\exists x_{W} \in W$ tal que $\forall z \in Z \quad a\left(x_{W}, z\right)=a(x, z)$;

b) $\left\|x-x_{W}\right\|_{X} \leq \frac{\|a\|}{\alpha_{1, z}} d_{X}(x, W)$,

onde $\alpha_{V, Z}=$ conorma $\left(\pi_{Z} A_{\mid V}\right)$ e $d_{X}(x, W)$ é dada pela expressão definida em (5).

\section{DEMONSTRAÇÃO.}

a) Este resultado é conseqüência imediata do Teorema de Lax-Milgram generalizado.

b) Para todo $z \in Z$. $\left(A\left(x-x_{W}\right), z\right)_{Y}=a\left(x-x_{W}, z\right)=0$. Logo $\pi_{Z} A\left(x-x_{W}\right)=0$, e se $v=\pi_{W}\left(x-x_{W}\right)$ e $\varpi=\pi_{W \perp}\left(x-x_{W}\right)=\pi_{W \perp} x$ então $\pi_{Z} A(v)=-\pi_{Z} A(\varpi)$. Daí deduzimos que:

$$
\alpha_{V, Z}\|v\|_{X} \leq\left\|\pi_{Z} A_{\mid V \perp}\right\|\|\varpi\|_{X}
$$

donde, aplicando o Teorema de Pitágoras, vem:

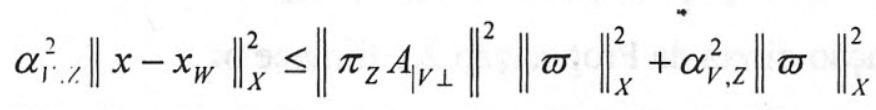

Por outro lado, o operador $\pi_{Z} A_{\mid W} \in L(W, Z)$ é sobrejetivo pois é $(W, Z) f$.c.. Logo, de acordo com o Lema 2.1, $\left\|\pi_{z} A_{\mid W \perp}\right\|^{2}+\alpha_{V, Z}^{2} \leq\left\|\pi_{Z} A\right\|^{2}$. Isto implica que

$$
\left\|x-x_{W}\right\|_{X} \leq \frac{\left\|\pi_{Z} A\right\|}{\alpha_{W, Z}}\|\varpi\|_{X} \leq \frac{\|A\|}{\alpha_{W, Z}}\|\varpi\|_{X}
$$

o que demonstra a Proposição, posto que $\varpi=\pi_{w \perp} x=x-\pi_{W} x$ ou seja $\|\varpi\|_{X}=d_{X}(x, W), \mathrm{e}\left\|\pi_{Z} A\right\| \leq\|A\|=\|a\|$.

\section{APROXIMAÇÃO ABSTRATA}

Apliquemos os resultados da seção anterior à aproximação de problemas variacionais lineares abstratos.

Quando se deseja aproximar a solução $x$ de um problema variacional definido sobre um par de espaços de Hilbert $(X, Y)$, o caminho natural a se percorrer é o segư⿱n亠te:

1. Verificar se $\left(P_{X .1}\right)$ está bem posto;

2. Construir dois subespaços $X_{h}$ e $Y_{h}$ de dimensão finita;

3. Verificar se $\left(P_{X_{h}, Y_{h}}\right)$ está bem posto; 
4. Resolver $\left(P_{X_{h}, Y_{h}}\right)$, de solução $x_{h} \in X_{h}$;

5. Majorar e estimar o erro $\left\|x-x_{h}\right\|_{X}$.

Os pontos 1. e 3. são assegurados pela fraca coercividade de $a$ sobre $X \times X$ e $X_{h} \times X_{h}$. Um procedimento usual para 2. é dado pelo método de Ritz, e assim 4. recai em usar algoritmos de resolução de sistemas lineares.

O objetivo desta Seção é tratar do último ponto 5., ou seja establecer estimativas destinadas a avaliar o erro ocorrido na aproximação do problema inicial, assim como a distância entre as soluções aproximadas obtidas com o uso de dois pares diferentes de subespaços. Tais aproximações são qualificadas de abstratas pois estabelecemos resultados em um quadro hilbertiano geral, exprimindo as estimativas em função de $d_{X}\left(x, X_{h}\right)$, sem procurar avaliar mais precisamente essa quantidade que, no caso de equações diferenciais por exemplo, pode ser estimada graças a resultados da teoria da interpolação nos espaços de Sobolev (cf. [11]).

Uma aplicação direta da Proposição 2.1 fornece o:

Teorema 3.1. Sejam $W$ e $Z$ subespaços fechados $X$ e $Y$, espaços de Hilbert, e $a \in L_{2 c}(X \times Y)$ que $\dot{e}(X, Y)$ f.c.. Então:

a) Para todo $L \in Y^{\prime}$, existe um único $\left(x, x_{W}\right) \in X \times Z$ tal que

$$
\forall y \in Y, a(x, y)=L(y) \text { e } \forall z \in Z, a\left(x_{W}, z\right)=L(z)
$$

b) Vale a mejoração do erro seguinte:

$$
\left\|x-x_{W}\right\|_{X} \leq \frac{\|a\|}{\alpha_{W, Z}} d_{X}(x, W)
$$

onde $x$ e $x_{W}$ são definidos em a).

DEMONSTRAÇÃO.

a) É uma consequéncia direta do Teorema 1.1.

b) Observa-se inicialmente que $\forall z \in Z, a\left(x_{W}, z\right)=a(x, z)$. Logo aplicando a Proposição 2.1 deduz-se imediatamente (11).

Esse s̉imples corolário da Proposição 2.1 é formulado como Teorema pois, conforme já antecipado na Seção 1, sob essa forma é uma generalização estrita da estimativa clássica (4) para problemas em que a forma a é coerciva, isto é, no caso presente (com $X=Y$ e $W=Z$ ): 


$$
\left\|x-x_{W}\right\|_{Y} \leq \frac{\|a\|}{\alpha} d_{Y}(x, Z),
$$

onde $\alpha$ denota a constante de coercividade de a sobre $Y \times Y$. Com efeito,

1. Não se requer a coercividade de $a$, mas somente a $(X, Y)$ fraca coercividade e a $(V, Z)$ fraca coercividade de $a$.

2. Mesmo no caso coercivo (com $X=Y$ e $W=Z$ ), a estimativa (11) é melhor do que (12), pois a constante que caracteriza a fraca coercividade é siempre maior do que a que figura na definição da coercividade. Além disso, esta última constante para um dado subespaço é sempre maior do que a relativa ao espaço inteiro.

Observação 3.1. Entretanto a fraca coercividade não fornece a estimativa melhorada do caso coercivo simétrico (cf. [8]):

$$
\left\|x-x_{W}\right\|_{Y} \leq\left(\frac{\|a\|}{\alpha}\right)^{\frac{1}{2}} d_{Y}\left(x_{W}, Z\right) .
$$

$\mathrm{Na}$ prática, usando por exemplo o Método dos Elementos Finitos clássico, temos $X=Y$, e utilizamos uma familia de subespaços $\left\{Y_{h}\right\}_{h}$ de dimensão finita, senḋo $h$ um parámetro ligado ao grau de refinamento de uma malha, à qual se associa cada subespaço de dimensão finita $Y_{h}$ destinado a aproximar $Y$ quando $h$ tende para 0 , e sobre os quais a é fracamente coerciva. Observa-se que se $a$ não for coerciva, assegurar a fraca coercividade de $a$ sobre toda a familia $\left\{Y_{h}\right\}_{h}, h>0$ pode ser um problema delicado.

Neste caso, chamando de $x_{h}$ a solução do problema variacional associado a um dado subespaço $Y_{h}$, a estimativa de erro (11) se escreve:

$$
\left\|x-x_{h}\right\|_{Y} \leq \frac{\|a\|}{\alpha_{h}} d_{Y}\left(x, Y_{h}\right),
$$

onde $\alpha_{h}$ é a conorma de $A_{h}=\pi_{Y_{h}} A_{Y_{h}}$. Logo, se $\alpha_{h}$ é independiente de $h$, o problema da aproximação de $x$ por $x_{h}$ é reduzido à capacidade de aproximação de $Y$ por $Y_{h}$, assunto já bem sedimentado na vasta literatura sobre o Método dos Elementos Finitos. Entretanto sugerimos na Seção seguinte, que essa teoria pode ser usada com a mesma eficácia para a análise de erro do Método de Volumes Finitos. 


\section{UM EXEMPLO NA APROXIMAÇÃO POR VOLUMES FINITOS}

Consideremos o seguinte problema:

Dado $f \in L^{2}(0,1)$, encontrar una função $u \in H^{1}(0,1)$ tal que,

$$
\left\{\begin{array}{l}
u^{\prime}+u=f \\
u(0)=0
\end{array}\right.
$$

O problema (13) pode ser colocado sob a forma variational abaixo:

$$
(P)\left\{\begin{array}{cc}
\text { Encontrar } u \in U & \text { tal que } \\
a(u, v)=L(v) & \forall v \in V .
\end{array}\right.
$$

onde

$$
\begin{gathered}
a(u, v)=\int_{0}^{1} u^{\prime} v d x+\int_{0}^{1} u v d x \\
V=L^{2}(0, L) \text { normado por }\|\cdot\|_{0,2}
\end{gathered}
$$

e

$$
U=\left\{v / v \in H^{1}(0, L), v(0)=0\right\} \text { normado por }\|\cdot\|_{1,2},
$$

sendo por definição,

$$
\|\cdot\|_{0,2}=\left[\int_{0}^{1}(\cdot)^{2} d x\right]^{1 / 2}
$$

$\mathrm{e}$

$$
\|\cdot\|_{1,2}=\left[\|\cdot\|_{0,2}^{2}+\left\|(\cdot)^{\prime}\right\|_{0,2}^{2}\right]^{1 / 2}
$$

Dado que tanto $V$ como $U$ são espaços de Hilbert, respectivamente munidos das normas dadas acima (cf. P.ex. [9]), para verificar que $(P)$ tem solução única para qualquer $f \in L^{2}(0,1)$. pode-se proceder da seguinte maneira:

Em primeiro lugar, dado $u \in U$ escolhemos $v=u^{\prime} \in V$. Claramente $a(u, v)=\left\|u^{\prime}\right\|_{0,2}^{2}+\int_{1}^{1} u u^{\prime} d x$, isto é:

$$
a(u, v)=\left\|u^{\prime}\right\|_{0,2}^{2}+u^{2}(1) / 2 \geq\left(1+C_{P}^{2}\right)^{-1}\|u\|_{1,2}^{2}
$$

onde $C_{P}$ é a constante da desigualdade de Poincaré que se aplica ao espaço $U$ (cf. [9]). Por otro lado, temos trivialmente,

$$
v\left\|_{0,2} \leq \mid u\right\|_{1,2}
$$

As relações (14) e (15) implicam que a condição (7) é satisfeita com $\alpha=\left(1+C_{P}^{2}\right)^{-1}$. 
Para verificar a condição (8), dado $v \in V$ não nulo, definimos $u \in U$ por $u(x)=\int_{0}^{x} v(t) d t, \quad x \in[0,1] . \quad$ Como $\quad u^{\prime}=v \neq 0$ por construção, temos $a(u, v)=\left\|u^{\prime}\right\|_{0,2}^{2}+\int_{0}^{1} u u^{\prime} d x \geq\|v\|_{0,2}^{2}$ e $\|u\|_{1,2} \leq\left(C_{P}^{2}+1\right)^{1 / 2}\|v\|_{0,2}$, o que verifica a condição desejada com uma constante igual a $\alpha^{1 / 2}$

Agora aproximamos $(P)$ pelo método dos volumes finitos do tipo centrado nos vértices, definido como se segue:

Inicialmente consideramos uma partição $T_{h}$ do dominio $(0,1)$ em volumes de controle $I_{i}$ com $i=1,2, \cdots, N$ de comprimentos iguais a $h=L / N$, sendo $N \in I^{*}$, onde $I_{i}([i-1] h, i h)$. Em seguida postulamos para aproximar $(P)$ :

$$
\left(P_{h}\right)\left\{\begin{array}{c}
\text { Encontrar } u_{h} \in U_{h} \quad \text { tal que } \\
a\left(u_{h}, v\right)=L(v) \quad \forall v \in V_{h} .
\end{array}\right.
$$

onde

$$
U_{h}=\left\{v / v \in C^{0}[0, L], v(0)=0, v_{/ I_{i}} \in P_{1} \quad \forall i, i=1,2, \cdots, N\right\}
$$

e

$$
V_{h}=\left\{v / v_{/ I_{i}} \in P_{0} \quad \forall i, i=1,2, \cdots, N .\right.
$$

sendo $P_{k}$ o espaço de polinómios de grau inferior ou igual a $k$.

O problema $\left(P_{h}\right)$ resume-se numa integração da equação diferencial de (13) em cada volume de controle, seguida pela substitução de $u$ por sua aproximação $u_{h} \in U_{h}$. Observe-se que o método assim definido pode também ser interpretado como um método dos elementos finitos conforme do tipo linear por pedaços usual, na medida em que $U_{h} \subset U$ e $V_{h} \subset V$.

Demonstra-se facilmente que o problema aproximado $\left(P_{h}\right)$ satisfaz as duas condições da forma (7) e (8). necessárias e suficientes para que ele tenha solução única $\forall f \in L^{2}(0, L)$. Para tanto pode-se usar exatamente os mesmos argumentos empregados acima para o caso do problema continuo $(P)$. De fato, basta levar em conta que a derivada de uma função de $U_{h}$ pertence a $V_{h}$, e reciprocamente a integral de 0 a $x$ de uma função de $V_{h}$ define necessariamente uma função de $U_{h}$. Assim sendo, conclui-se que no caso do problema aproximado a condição (7) é verificada $\operatorname{com} \alpha_{h}=\alpha$. 
Agora se em $(P)$ consideramos o caso em que $f \in H^{1}(0, L)$, pode-se verificar facilmente que $u \in H^{2}(0, L)$ (ou seja $u^{\prime \prime} \in L^{2}(0,1)$ ). Neste caso, usando os resultados clássicos de aproximação polinomial por pedaços (cf. [11]), deduzimos que existe uma constante $C$ independiente de $h$, tal que:

$$
d_{U}\left(u, U_{h}\right) \leq C h\left\|u^{\prime \prime}\right\|_{0,2} .
$$

Daí se conclui imediatamente de (11) que

$$
\left\|u-u_{h}\right\|_{1,2} \leq \frac{C \sqrt{2}}{\alpha_{h}} h\left\|u^{\prime \prime}\right\|_{0,2} .
$$

A análise desenvolvida acima - tipicamente empregada na literatura para métodos de elementos finitos -, pode ser estendida a outros esquemas de tipo volumes finitos, em uma ou mais dimensões técnicas de certa dificuldade. A esse respeito pode-se citar alguns trabalhos recentes, entre os quais por exemplo, o de BARANGER e MAÎTRE [4]. Na realidade estamos trabalhando para apresentar no futuro próximo alguns outros exemplos que ilustram essa asserção, pois acreditamos que estudos nessa linha contribuam para desmitificar um pouco mais as por vezes tão decantadas diferenças entre as abordagens de elementos finitos e de volumes finitos.

\section{REFERÊNCIAS}

1. AUBIN, J.P., Approximation of Elliptic Boundary-Value Problems, Wiley, New York, (1972).

2. AZIZ, A.K. \& BABUSKA, I., Survey Lectures on the Mathematical Foundations of the Finite Element Method with Applications, em: The Mathematical Foundations of the Finite Element Method with Applications to Partial Differential Equations, A.K. Aziz ed., Academic Press, New York, (1972).

3. BABUSKA, I., Error Bound for the Finite Element Method, Numerische Mathematik, 16 (1971), 322 333.

4. BARANGER, J. \& MAÎTRE, J.F., a sair.

5. BRAMBLE, J.H. \& ZLAMAL, M., Triangular Elements in the Finite Element Method, Mathematics of Computation, 24, 112 (1970), 809-820.

6. CIARLET, P.G. \& RAVIART, P.A., General Lagrange and Hermite Interpolation in IR $^{\mathrm{n}}$ with Application to the Finite Element Method. Archives of Rational Mechanics Anal., 46 (1972), 177-199.

7. DUPIRE, B., Problemas Variacionais Lineares, sua Aproximação e Formulações Mista, tese de duotorado, Depto. Informática, Pontificia Universidade Católica do Río de Janeiro, (1985).

8. RAVIÂRT, P.A. \& THOMAS, J.M., Introduction à analyse numérique des équations aux derivées partielles, Masson, Paris. (1983).

9. RUAS, V., Uma Introdução aos Problemas Variacionais, Ed. Guanabara 2, Río de Janeiro, (1979).

10. STRANG, G., Variational Crimes in the Finite Element Method, in: The Mathematical Foundations of the Finite Element Method with Applications to Partial Differential Equations, A.K. AZIZ ed., Academic Press, New York, (1972).

11. STRANG, G. \& FIX, G.. An Analysis of the Finite Element Method, Prentice Hall, New York, (1973). 